\title{
Electrostatic Properties of Molecules from the X-Ray Charge Density. Application to Deuterated Benzene, l-Alanine and d,l-Histidine *
}

\author{
Zhengwei Su and Philip Coppens \\ Chemistry Department, State University of New York at Buffalo, Buffalo, NY 14214, USA
}

Z. Naturforsch. 48a, 85-90 (1993); received April 23, 1992

It has been shown (Z. Su and P. Coppens, Acta Cryst. A 48, 188 (1992)) that the electrostatic potential, the electric field, and the electric field gradient (EFG) can be expressed in closed forms in terms of the positions and the charge-density parameters of individual atoms, whose aspherical density is described by a pseudoatom model (e.g., N. Hansen and P. Coppens, Acta Cryst. A 34, 909 (1978)). A FORTRAN program MOLPROP 91 based on this method has been written (Z. Su, State Univ. of New York at Buffalo 1991). The method has been applied to the title compounds. Low-temperature X-ray diffraction data of fully deuterated benzene (G. A. Jeffrey, J. R. Ruble, Y. Yeon, and C. Lemann, private communication, 1991), l-alanine (R. Destro, R. E. Marsh, and R. Bianchi, J. Phys. Chem. 92, 966 (1988) and $d, l$-histidine (N. Li, Ph.D. thesis, State University of New York at Buffalo 1989) were analyzed using the least-squares deformation density refinement program LSMOL 90 (a modified version of MOLLY). MOLPROP 91 was subsequently used to calculate the electrostatic-potential maps in selected sections, and at the nuclear positions. For the latter, the EFGs were also evaluated. The electrostatic potentials were used to fit net atomic charges and estimate the molecular energies. Errors in the derived quantities are given.

Key words: Electron density; Electrostatic potential; Electric field; EFG; Molecular energy.

\section{Introduction}

Physical properties calculated from the X-ray charge density can be compared with more directly measured values to serve as a test of the accuracy of the charge density. Conversely, physical properties can be evaluated from the experimental density to provide information about the chemical bonding and chemical reactivities. Among these properties are the electrostatic potential, the electric field and the electric field gradient. We outline here a method to analytically evaluate these properties from the multipole description of the charge density, and apply the method to the title compounds.

Furthermore, in order to obtain more quantitative and compact information about the charge density, fitted net atomic charges and estimated molecular energies have been derived.

We note that several results for the electrostatic potential and the electric field gradient have been pub-

* Presented at the Sagamore X Conference on Charge, Spin and Momentum Densities, Konstanz, Fed. Rep. of Germany, September 1-7, 1991.

Reprint requests to Prof. Dr. Philip Coppens, Crystallography Laboratory, Department of Chemistry, Faculty of Natural Sciences and Mathematics, University at Buffalo (State University of New York), Acheson Hall, Buffalo, New York 14214, USA. lished (see, e.g., [1-5]). However, we have not been able to compare the formalisms presented here with those used earlier, as no record appears to be available in the literature.

\section{The Method of Calculation}

We consider the pseudoatom model [6] in which the aspherical electronic density of an atom is described as

$$
\begin{aligned}
\varrho_{\mathrm{e}}(r)= & P_{\mathrm{c}} \varrho_{\mathrm{c}}(r)+P_{\mathrm{v}}{\chi^{\prime}}^{3} \varrho_{\mathrm{v}}\left(\varkappa^{\prime} r\right) \\
& +\sum_{l=0}^{l_{\max }} \chi^{\prime \prime 3} R_{l}\left(\chi^{\prime \prime} r\right) \sum_{m=0}^{l} \sum_{p} P_{l m p} d_{l m p}(\theta, \phi),
\end{aligned}
$$

where the radial dependence of the density components is described by Slater-type functions (single exponential functions) or linear combinations of STFs, and the angular dependence is given by surface harmonic functions [7], and where $\varrho_{c}(r)$ and $\varrho_{v}(r)$ are coreand valence-electron densities constructed from the canonical Hartree-Fock atomic orbitals, $R_{l}(r)$ is a normalized Slater-type radial function (STF), and $P_{\mathrm{c}}, P_{\mathrm{v}}$ and $P_{l m p}$ are the population coefficients. $p$ can be + or - for nonzero $\operatorname{l.~}_{l_{\text {lmp }}}(\theta, \phi)$ are normalized real spherical harmonic functions.

In the following discussion we are concerned with a single molecule extracted from the crystal, with the 
thermal-motion effect excluded. From the principle of superposition, the electrostatic properties represented in integral form are (see Fig. 1 for definitions of the vectors)

$$
\begin{aligned}
\Phi\left(\boldsymbol{R}_{\mathrm{p}}\right) & =\int \frac{\varrho_{\mathrm{t}}(r)}{\left|\boldsymbol{R}_{\mathrm{p}}-\boldsymbol{r}\right|} \mathrm{d} \boldsymbol{r} \\
\boldsymbol{E}\left(\boldsymbol{R}_{\mathrm{p}}\right) & =\int \frac{\left(\boldsymbol{R}_{\mathrm{p}}-\boldsymbol{r}\right) \varrho_{\mathrm{t}}(r)}{\left|\boldsymbol{R}_{\mathrm{p}}-\boldsymbol{r}\right|^{3}} \mathrm{~d} \boldsymbol{r} \\
\nabla \boldsymbol{E}_{m n}\left(\boldsymbol{R}_{p}\right) & =-\int \frac{3 x_{m} x_{n}-\delta_{m n}\left(\left|\boldsymbol{R}_{p}-\boldsymbol{r}\right|\right)^{2}}{\left|\boldsymbol{R}_{p}-\boldsymbol{r}\right|^{5}} \varrho_{\mathrm{t}}(\boldsymbol{r}) \mathrm{d} \boldsymbol{r},
\end{aligned}
$$

where $\varrho_{\mathrm{t}}(\boldsymbol{r})=\varrho_{\mathrm{n}}(\boldsymbol{r})-\varrho_{\mathrm{e}}(\boldsymbol{r})$ is the total charge density, the subscripts $\mathrm{n}$ and $\mathrm{e}$ denoting the nuclear and electronic densities, respectively. $x_{k}$ is the $k$-th component of $\boldsymbol{R}_{p}-\boldsymbol{r}$ in a Cartesian coordinate system and $\delta$ is the Kronecker delta.

There are two classes of contributions to these properties, depending on whether or not the field point is at a nuclear site:

a) Central and peripheral contributions for points at atomic sites.

b) Peripheral contributions only at general points.

The central contributions are given by one-electron one-center integrals. Owing to the orthonormality of the surface spherical harmonic functions, only the spherical density (monopoles) makes a central contribution to the electrostatic potential, only the dipoles make contributions to the electric field, and only the quadrupoles to the electric field gradient.

Following the notations of [7], we have the central contribution owing to a monopolar density centered at the parent atom as

$$
-P_{\mathrm{M}, 00} \frac{x^{\prime \prime} \zeta_{0}}{\left(n_{0}+2\right)} \text {. }
$$

The central contribution to the $x$-component of the electric field owing to a dipole density is

$$
-\frac{4}{3} P_{\mathrm{M}, 11+} \frac{\left(\varkappa^{\prime \prime} \zeta_{1}\right)^{2}}{\left(n_{1}+1\right)\left(n_{1}+2\right)}
$$

and the $z z$ element of the electric field gradient tensor is

$$
\frac{6}{5} \frac{\left(\varkappa^{\prime \prime} \zeta_{2}\right)^{3}}{n_{2}\left(n_{2}+1\right)\left(n_{2}+2\right)} \sqrt{3} P_{\mathrm{M}, 20} .
$$

Note that the quantities in (5), (6), and (7) are referred to the coordinate system with respect to which the

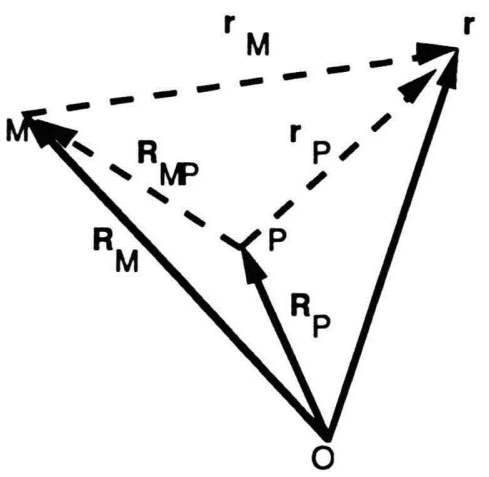

Fig. 1. Definitions of vectors used in the description of the electrostatic functions.

spherical harmonic functions, and thus the population coefficients, are defined.

The peripheral contributions are due to the neighboring atomic densities and are given by one-electron two-center integrals. In the derivation, the Fourier Convolution Theorem Method [8] is applied to evaluate the integrals. The derivation and the resulting expressions are given in [7]. A FORTRAN program MOLPROP 91 based on this method has been written [9].

The leading term at long separation of the source point and the field point is the point-charge-like term; the remaining terms decay faster the more compact the density is (larger $\zeta)$.

\section{Applications of the Method}

\section{a) The Electrostatic Potentials}

Details of refinements of the data sets were discussed in $[7,10]$.

Figures 2, 3, and 4 are the electrostatic potentials and their estimated standard deviations in the selected planes.

The electrostatic potential is mainly due to the monopolar densities. For points about $3 \AA$ away from all nuclei, the point-charge approximation is justifiable.

The analytical expressions of the electrostatic potential as a function of the positional and charge-density parameters also suggest that the main source of error is due to the errors in the multipole population parameters at least at points along the periphery of the molecule. Hence the estimated standard-deviation-contour plots are calculated from the esds of these 

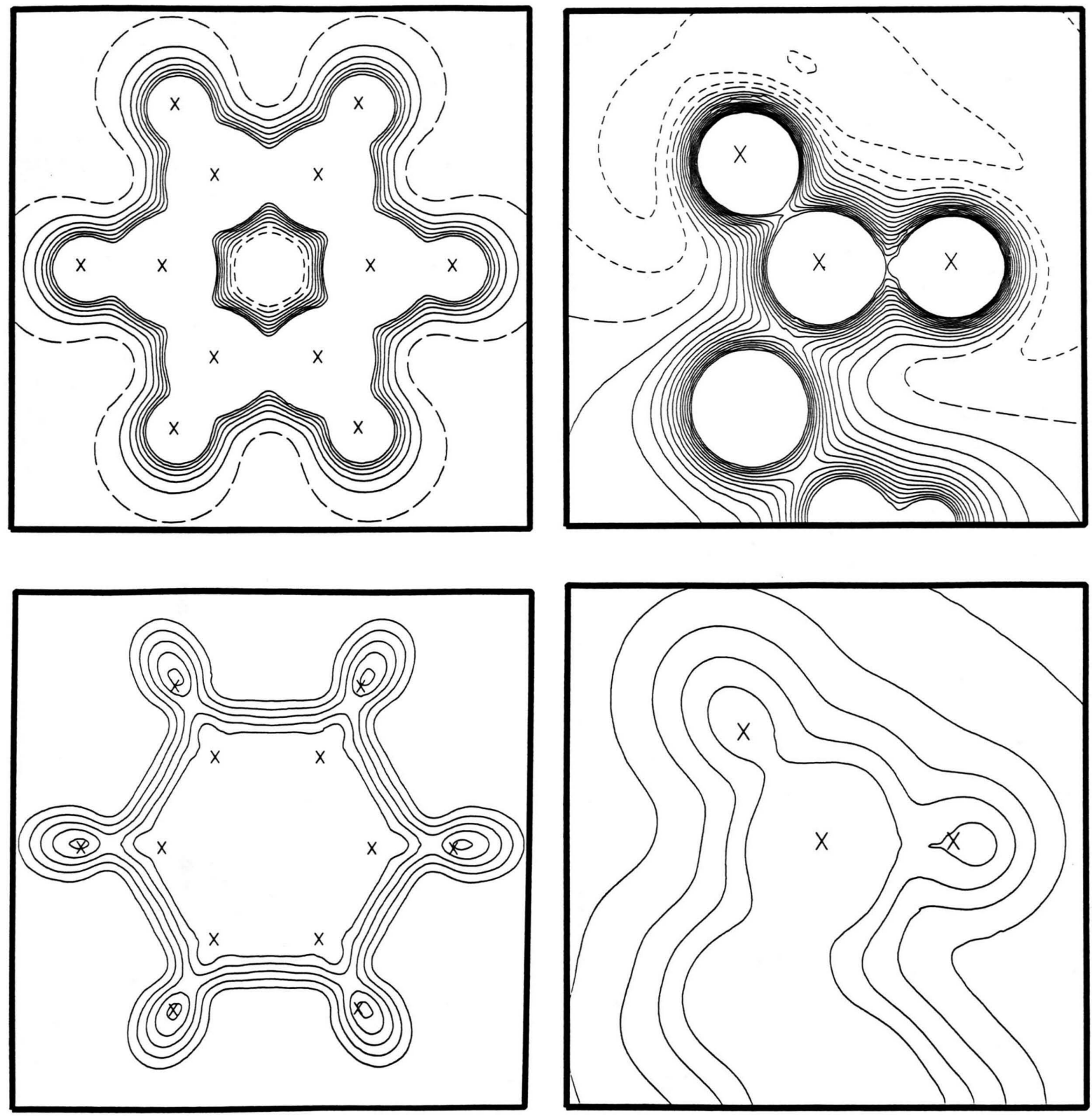

Fig. 2. Electrostatic-potential contour plot for $\mathrm{C}_{6} \mathrm{D}_{6}$ from a single extracted molecule. Sections of $7 \AA \times 7 \AA$ size in the $\mathrm{C}_{6} \mathrm{D}_{6}$ least-squares plane are shown. Negative contours: short dashed lines. Zero contours: long dashed lines. Positive contours: solid lines. a) Potential due to the total charge density. Minimum contour $-0.02 \mathrm{e} / \AA$; maximum contour $0.68 \mathrm{e} / \AA$. Contour intervals at $0.10 \mathrm{e} / \AA$. b) Estimated standard deviations of the potential. Minimum contour $0.010 \mathrm{e} / \AA$; maximum contour $0.018 \mathrm{e} / \AA$. Contour intervals at $0.002 \mathrm{e} / \AA$.

Fig. 3. Electrostatic-potential contour plot for $l$-alanine from a single extracted molecule. Section of $5 \AA \times 5 \AA$ size in the COO least-squares plane are shown. Negative contours: short dashed lines. Zero contours: long dashed lines. Positive contours: solid lines. a) Potential due to the total charge density. Minimum contour $-0.45 \mathrm{e} / \AA$; maximum contour $1.95 \mathrm{e} / \AA$. Contour intervals at $0.15 \mathrm{e} / \AA$. b) Estimated standard deviations of the potential. Minimum contour $0.010 \mathrm{e} / \AA$; maximum contour $0.050 \mathrm{e} / \AA$. Contour intervals at $0.01 \mathrm{e} / \AA$. 

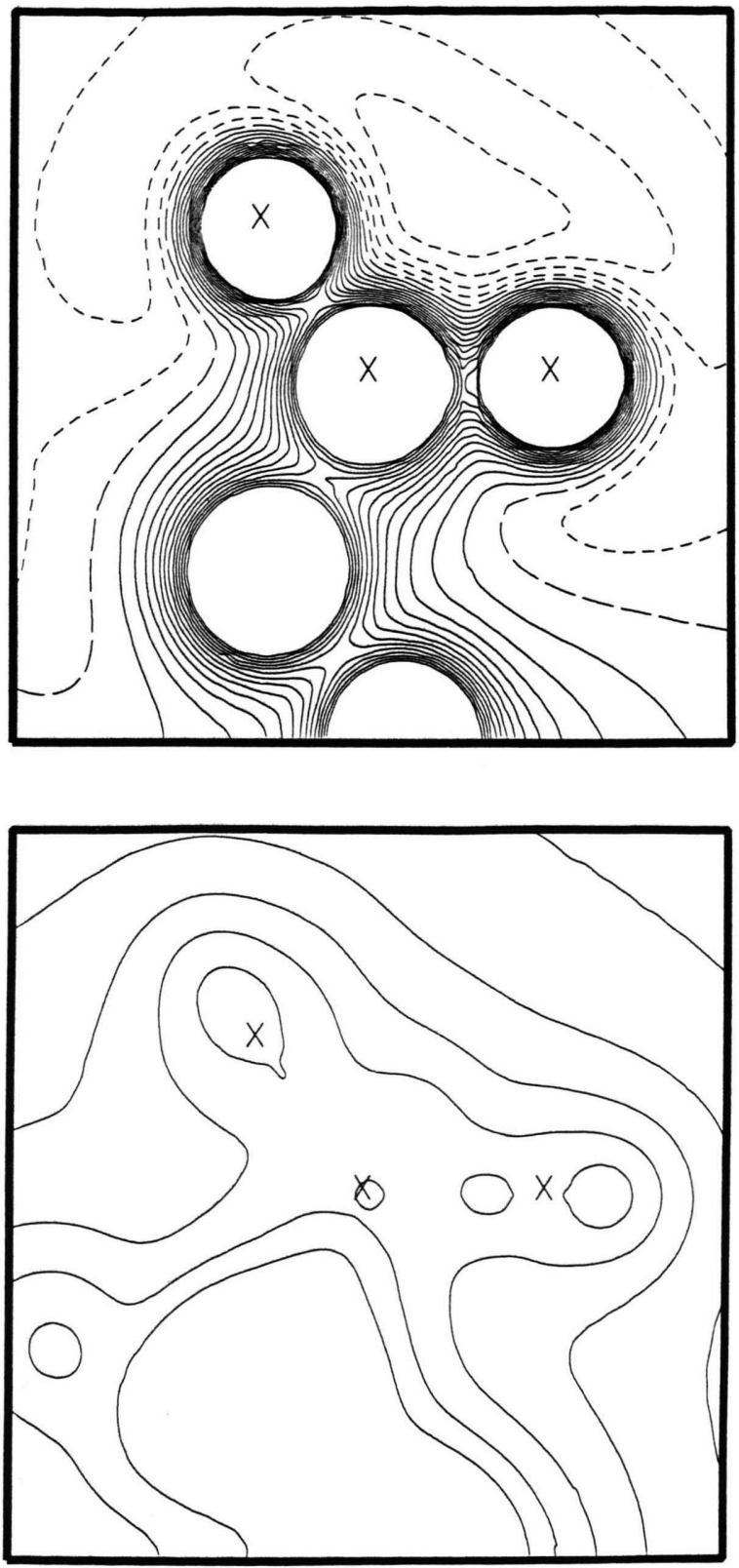

Fig. 4. Electrostatic-potential contour plot for $d, l$-histidine from a single extracted molecule. Sections of size $5 \AA \times 5 \AA$ through the COO least-square plane are shown. Negative contours: short dashed lines. Zero contours: long dashed lines. Positive contours: solid lines. a) Potential due to the total charge density. Minimum contour $-0.60 \mathrm{e} / \AA$; maximum contour $1.95 \mathrm{e} / \AA$. Contour intervals at $0.15 \mathrm{e} / \AA$. b) Estimated standard deviations of the potentials. Minimum contour $0.010 \mathrm{e} / \AA$; maximum contour $0.080 \mathrm{e} / \AA$. Contour intervals at $0.01 \mathrm{e} / \AA$
Table 1. Total energies from the X-ray charge density.

$\begin{array}{ll}\text { Benzene [13] } & \\ \text { Total energy } & \Sigma \text { (isolated atoms) } \\ -237.5(6) & -229.1\end{array}$

Comparison with ab initio HF-SCF results (Gaussian 88 [14])

$\begin{array}{lll}6-31 G^{* *} & \begin{array}{ll}\text { From } \\ \text { potentials }\end{array} & \text { MP2 } \\ -230.7 & -230.1 & -231.5\end{array}$

\section{d,l-Histidine [10]}

$\begin{array}{llll}\text { Total } & \text { STO-3G } & \sum \text { (isolated atoms) } \\ -557(3) & -538.4 & -543.5 & \\ & & & \\ \text { l-Alanine [15] } & & & \\ \text { Total } & \text { STO-3G } & 6-31 \mathrm{G}^{* *} & \Sigma \text { (isolated atoms) } \\ -329(3) & -317.5 & -321.8 & -320.1\end{array}$

Table 2. Electric field gradients (EFG) (a.u.) at nuclear positions. $x x$, etc. represents the tensor element $\nabla E_{x x}$, etc. $\eta$ is the asymmetry parameter, QCC is the quadrupole coupling constant $e^{2} Q \nabla E_{z z} / h$.

$\begin{array}{lrcc}\text { Deuterons in } C_{6} D_{6}[13] & & \\ & \text { X-ray } & \text { NMR [16] } & 6-31 G^{* *} \\ x x & 0.16(2) & 0.143 & 0.181 \\ y y & 0.13(2) & 0.132 & 0.157 \\ z z & -0.29(2) & -0.275 & -0.338 \\ \eta & 0.10(7) & 0.04 & 0.07\end{array}$

$N(1)^{\text {a }}$ in d,l-histidine [10]

$\begin{array}{lll} & \text { X-ray } & \text { STO-3G } \\ x x & 0.23(3) & -0.18 \\ y y & 0.02(2) & -0.09 \\ z z & 0.25(10) & 0.27 \\ \eta & 0.8(7) & 0.31\end{array}$

$N$ in l-alanine [15]

\begin{tabular}{lcc} 
& X-ray & NQR [17] \\
$x x$ & $0.05(2)$ & \\
$y y$ & $0.08(3)$ & \\
$z z$ & $-0.13(3)$ & \\
$\eta$ & $0.25(40)$ & \\
QCC $(\mathrm{MHz})$ & $-0.5(2)$ & + or -1.2 \\
\hline
\end{tabular}

a $\mathrm{N}(1)$ is nitrogen in $\mathrm{NH}_{3}^{+}$group. 
Table 3. Net atomic charges from the fit to experimental potentials.

\begin{tabular}{|c|c|c|c|c|c|c|c|}
\hline & CHELP & $\begin{array}{l}\text { Monopoles } \\
\text { aspherical atom } \\
\text { refinement }\end{array}$ & $\begin{array}{l}x \text { refine- } \\
\text { ment }\end{array}$ & STO-3G & $3-21 \mathrm{G}$ & 6-31G & $6-31 \mathrm{G}^{* *}$ \\
\hline \multicolumn{8}{|c|}{$C$ in deuterated benzene [13]. Residual index 0.08.} \\
\hline & $-0.27(3)$ & $-0.21(3)$ & $-0.15(1)$ & -0.062 & -0.24 & -0.20 & -0.147 \\
\hline \multicolumn{8}{|c|}{$\mathrm{C}(1), \mathrm{O}(1), \mathrm{O}(2)$ in $\mathrm{COO}^{-}$group of d,l-histidine [10]. Residual index 0.25.} \\
\hline $\begin{array}{l}\mathrm{C}(1) \\
\mathrm{O}(1) \\
\mathrm{O}(2)\end{array}$ & $\begin{array}{r}0.11 \\
-0.68 \\
-0.65\end{array}$ & $\begin{array}{l}-0.14(7) \\
-0.43(4) \\
-0.47(4)\end{array}$ & $\begin{array}{r}0.51(6) \\
-0.65(4) \\
-0.68(4)\end{array}$ & $\begin{array}{r}0.22 \\
-0.48 \\
-0.44\end{array}$ & & & \\
\hline \multicolumn{8}{|c|}{$\mathrm{C}(1), \mathrm{O}(1), \mathrm{O}(2)$ in $\mathrm{COO}^{-}$group of l-alanine [15]. Residual index 0.09.} \\
\hline $\begin{array}{l}\mathrm{C}(1) \\
\mathrm{O}(1) \\
\mathrm{O}(2)\end{array}$ & $\begin{array}{r}0.55 \\
-0.65 \\
-0.76\end{array}$ & $\begin{array}{l}0.23(10) \\
-0.37(4) \\
-0.45(4)\end{array}$ & $\begin{array}{r}0.49 \\
-0.58 \\
-0.69\end{array}$ & $\begin{array}{r}0.22 \\
-0.48 \\
-0.44\end{array}$ & & & $\begin{array}{r}0.23 \\
-0.72 \\
-0.72\end{array}$ \\
\hline
\end{tabular}

parameters. As may be expected, the errors become larger for points closer to a nucleus.

\section{b) Total Molecular Energy from the Electrostatic Potentials at the Nuclei and Electric-Field-Gradient Calculations}

A well documented formula owing to Politzer relates the approximate molecular energy to the electrostatic potentials at atomic nuclei [11]:

$$
\sum_{\text {atom } i}^{\text {all atoms }} k_{i} Z_{i} \Phi_{i}(0) \text {. }
$$

$k_{i}$ is taken as $3 / 7$, or can be derived from the HartreeFock atomic energies, or chosen so that the homonuclear diatomics have the exact Hartree-Fock energies [12].

We have applied (8) to the title compounds, using the electrostatic potentials calculated from the pseudoatom description of the charge density and take the coefficient $k_{i}$ as $3 / 7$. Results are listed in Table 1 . The energies are in a.u. (hartrees) $\left(1 E_{\mathrm{h}}=27.2 \mathrm{eV}\right)$.

The results do not change significantly when $k_{i}$ optimized for atoms are used instead of the value $3 / 7$.

Results on electric field gradient are listed in Table 2.

\section{c) Atomic Charges Derived from Electrostatic Potentials}

The program CHELP [18] has been adapted to derive the point atomic charges at the atomic positions that give the best fit to the electrostatic potentials at selected points. The method is a constrained least-squares fit procedure using Lagrange multipliers. In the present work, only the molecular neutrality is constrained. Points on a cubic grid are used in the fitting procedure; points within the van der Waals radius of any of the atoms were excluded. The residual index is defined as

$$
R=\frac{\sum_{i=1}^{N_{\mathrm{p}}}\left|V_{1}(i)-V_{2}(i)\right|}{\sum_{i=1}^{N_{\mathrm{p}}}\left|V_{1}(i)\right|},
$$

where $N_{\mathrm{p}}$ is the number of points used for the fitting, $V_{1}(i)$ and $V_{2}(i)$ are the electrostatic potentials calculated from the pseudoatom model and the pointcharge model, respectively. $N_{\mathrm{p}}$ is chosen to be greater than 100 times the number of atoms in the molecule. Results are listed in Table 3.

The fitted charges do not always agree well with the Mulliken charges or net experimental charges from the monopole populations, but with the $1 \AA$ cubic grid used, they seem relatively independent of the exact positions of the grid points. For benzene, for example, a different orientation of the grid axes changed the result by less than $0.01 \mathrm{e}$. We note that a random point location methodology for the charge-fitting procedure has been discussed by Woods et al. [19].

To summarize, we have shown that within the limits of the model used to describe the charge density, the electrostatic properties are readily expressed in closed form. The approximate molecular energies derived from the electrostatic potentials at the atomic nuclei seem to be systematically too negative. The EFG ten- 
sors at the deuterons in fully deuterated benzene agree very well with the spectroscopic and theoretical results. EFGs at $\mathbf{N}$ in $l$-alanine and $d, l$-histidine show larger deviations. The reasons may be the lack of Sternheimer shielding and anti-shielding corrections and the inadequacy of the density model in the regions very close to the nuclei. The atomic point charges from fitting the calculated electrostatic potentials are useful in the calculations of electrostatic interactions, but more experience should be obtained in their application.

[1] M. Spackman and R. F. Stewart, in: Chemical Applications of Atomic and Molecular Electrostatic Potentials (P. Politzer and D. G. Truhlar, eds.), Plenum Press, New York 1981.

[2] S. Swaminathan and B. M. Craven, Acta Cryst. B 40, 511 (1984).

[3] X. M. He, S. Swaminathan, B. M. Craven, and R. K. McMullan, Acta Cryst. B 44, 271 (1988).

[4] R. Destro, R. Bianchi, and G. Morosi, J. Phys. Chem. 93, 4447 (1989).

[5] R. F. Stewart, in: Applications of Charge Density Research to Chemistry and Drug Design (G. A. Jeffrey and J. F. Piniella, eds.), Plenum Press, New York 1991.

[6] N. Hansen and P. Coppens, Acta Cryst. A 34, 909 (1978).

[7] Z. Su and P. Coppens, Acta Cryst. A 48, 188 (1992)

[8] F. P. Prosser and C. H. Blanchard, J. Chem. Phys. 36, 1112 (1962).

[9] Z. Su, Molprop 91. Experimental Molecular Electrostatic Properties. State University of New York at Buffalo, New York, USA 1991.

\section{Acknowledgements}

We would like to thank Dr. M. Francl of Bryn Mawr College for making the program CHelP available, Dr. Ruble of the University of Pittsburgh for supplying the data on deuterated benzene, and Dr. Jiali Gao of the State University of New York at Buffalo for use of the STARDENT computer. Support of this work by the National Science Foundation (CHE9021069) is gratefully acknowledged.

[10] N. Li, Ph.D. thesis, State University of New York at Buffalo, New York 1989.

[11] P. Politzer, J. Chem. Phys. 70, 1067 (1979).

[12] T. Anno, J. Chem. Phys. 72, 782 (1980).

[13] G. A. Jeffrey, J. R. Ruble, Y. Yeon, and C. Lemann, private communication 1991.

[14] Gaussian 88. M. J. Frisch, M. Head-Gordon, H. B. Schlegel, K. Raghavachari, J. S. Binkley, C. Gonzalez, D. J. Defrees, D. J. Fox, R. A. Whiteside, R. Seeger, C. F. Melius, J. Baker, R. L. Martin, L. R. Kahn, J. J. P. Stewart, E. M. Fluder, S. Topiol, and J. A. Pople, Gaussian, Inc., Pittsburgh, PA 1988.

[15] R. Destro, R. E. Marsh, and R. Bianchi, J. Phys. Chem. 92, 966 (1988).

[16] F. S. Millett and B. P. Dailey, J. Chem. Phys. 56, 3249 (1972).

[17] M. J. Hunt, J. Magn. Reson. 15, 113 (1974).

[18] L. E. Chirlian and M. M. Francl, J. Comp. Chem. 8, 894 (1987).

[19] R. J. Woods, M. Khalil, W. Pell, S. H. Moffat, and V. H. Smith, Jr., J. Comp. Chem. 11, 297 (1990). 